\title{
Corners, receptive fields, and visually evoked cortical potentials*
}

\author{
ANNE F. MOSKOWITZ, JOHN C. ARMINGTON, and GEORGE TIMBERLAKE \\ Northeastern University, Boston, Massachusetts 02115
}

\begin{abstract}
Alternating stimuli with herringbone patterns were used to obtain visually evoked cortical potentials (VECPs) from three human Ss. Two sets of stimulus patterns were used, one with sharp corners and one with the corners rounded off. Each set ranged in angularity from 180 to 45 deg in 45-deg steps. Results showed that: (a) VECP response amplitude was greatest for the 90-deg-corner pattern, (b) response amplitude was greater for the 45-deg-corner pattern than for the 135-deg-corner pattern, and (c) cornered and rounded patterns evoked responses of greater amplitude than those evoked by the straight (180-deg) patterns. Also, the peak latency of responses to cornered patterns was shorter than that of responses to rounded and straight patterns.
\end{abstract}

Visually evoked cortical responses to spatially unstructured (blank) stimuli are consistently smaller in amplitude than responses to spatially structured (patterned) stimuli (Spehlman, 1965; Spekreijse, 1966). Several studies have examined either the amplitude of specific components or the overall amplitude of visually evoked cortical responses as a function of pattern configuration, spatial frequency, and orientation of pattern elements. Spehlman (1965), White (1969), and Armington, Corwin, and Marsetta (1971) have all demonstrated that a checkerboard pattern generates a larger response than a stripe pattern. It has been suggested that the presence of contrast borders, or edges (Spekreijse, 1966), the number of contrast borders (Spehlman, 1965), the total length of contrast borders (Reitveld, Tordoir, Hagenouw, Lubbers, \& Spoor, 1967), the number of pattern elements (Regan, 1972), and contour sharpness (Harter \& White, 1968) partially account for differences in responses generated by patterned and unpatterned stimuli and by differing patterned stimuli. Armington, Corwin, and Marsetta (1971) use a simple model based on receptive fields with circular spatially antagonistic center-surround organizations to account for the larger response amplitude to checks than to stripes of intermediate spatial frequencies; a check pattern allows less light to fall on the inhibitory surround than does a stripe pattern of the same spatial frequency. Timberlake (1972) found evidence for greater evoked potential amplitude due to the presence of corners and also used the center-surround receptive field model to explain his results. Reitveld et al (1967) suggest that the presence and angularity of intersecting borders (corners) have an important effect on evoked potential amplitude.

An additional stimulus dimension which has been found to affect the visually evoked cortical potential is the orientation of pattern elements. Building upon

*This research was supported by Public Health Service Grant EY00568. psychophysical data and upon evoked potential data, respectively, Campbell and Kulikowski (1966) and Campbell and Maffei (1970) suggested that there were neural "orientation detectors" in the human visual system. Their work is consistent with descriptions by Hubel and Wiesel $(1962,1965)$ and Campbell, Cleland, Cooper, and Enroth-Cugell (1968) of orientational selectivity of single units in the cat and monkey cortex.

The purpose of the present study is to investigate the effect on the visually evoked response of (a) the presence of corners, (b) the angularity of corners, and (c) the orientation of pattern elements, using the method of stimulus alternation (Johnson, Riggs, \& Schick, 1966). While other investigators have attempted to explain effects due to such variables on the basis of complex and hypercomplex cortical receptive fields, we will try to account for our findings in terms of simple retinal receptive fields.

\section{METHOD}

Alternating stimuli were presented to the S's right eye in Maxwellian view (Westheimer, 1966) by means of a stimulator similar to that described by Armington (1968). The optical system is schematically represented in Fig. 1. A $2 \mathrm{~Hz}$ square-wave pulse applied to an electric armature caused the pivoted mirror to move back and forth, resulting in a lateral displacement of the pattern across the final field stop every $250 \mathrm{msec}$ : Pulse voltage was adjusted so that the excursion equaled the wid th of a pattern element; thus, the dark and light areas of the pattern were interchanged with each movement. Each change in stimulation elicited a small local response, a preset number of which were cumulated by an averaging computer. There was no change in the light flux entering the eye, since the total light and dark areas remained constant during both halves of the displacement cycle. Thus, the amount of stray light remained constant throughout. The use of periodic stimulus presentation insures that adaptation is held constant. Preliminary data indicated that different stimulation rates, up to $8 \mathrm{~Hz}$, produced similar results, in agreement with Spekreijse (1966), who found that the evoked potential amplitude vs stimulus frequency curve is flat up to about 20-30 reversals/sec. An alternation rate of $2 \mathrm{~Hz}$ was chosen because, according to Regan 


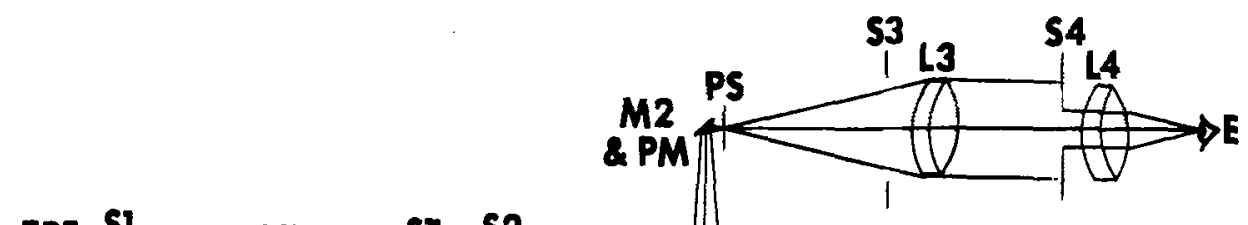

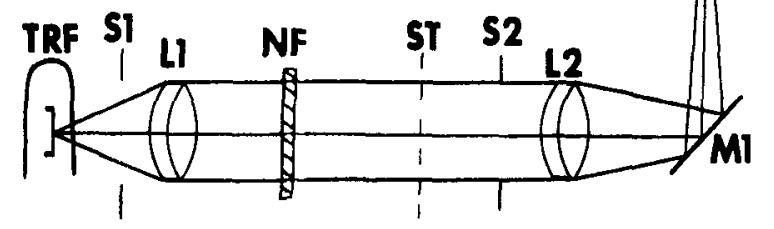

(1972), for frequencies of pattern reversal of 4 or fewer reversals per second, each reversal generates a separate response.

Visually evoked cortical potentials were recorded monopolarly, using silver cup electrodes. The active electrode was attached to the scalp over the inion, the reference electrode was at the right earlobe, and the ground electrode was at the left earlobe. This midline electrode placement is consistent with our earlier work and was chosen because cortical representation of foveal projections is medially located (Holmes, 1918). Gastaut and Regis's (1964) topographic analysis of the visually evoked potential indicated that the electrode placement which gave maximum amplitude and precision was on the inion. The electrodes were led through two resistance-capacitance amplifiers connected in cascade with a total gain of $10^{5}$ and a bandpass from 0.2 to $50 \mathrm{~Hz}$. The output was taken to an average response computer. The complete analysis cycle was $500 \mathrm{msec}$; with a response occurring every $250 \mathrm{msec}$. Thus, each record showed two averaged responses.

The eight stimulus patterns are shown as the $S$ viewed them in Fig. 2. Each bar subtended 0.5 deg of visual angle. The patterns fell within a 5-deg diameter when moving laterally. They were presented against a homogeneously illuminated 412-troland circular field, $5 \mathrm{deg}$ in diam. Corresponding angular cornered and rounded pattern pairs (Fig. 2: 2 and 6,3 and 7 , and 4 and 8 ) were identical in all respects except for the lack of sharp corners in the rounded patterns. The two straight line patterns (Fig. 2: 1 and 5) were identical.

The four patterns within each set differed from each other in two major aspects: (a) angularity of central corners or curves, and concomitantly, (b) orientation of the line segments which comprised the pattern elements. The three cornered patterns were equated for number of central corners (6). All eight patterns were equated for amount of edge (25.5 deg of visual angle), number of pattern elements (6), number of noncentral intersecting borders (12),' spatial frequency $(1.0$ cycle per degree), retinal illuminance of the transparent areas (412 trolands), retinal illuminance of the opaque areas (17 trolands), and contrast between light and dark areas (92.1\%). 1 Pilot data indicated that increasing (or decreasing) retinal illuminance and contrast yielded an overall increase (or decrease) in evoked potential amplitude, affecting all eight stimulus patterns to a proportionately equal degree.

A standard checkerboard pattern with a check width of 0.5 deg of visual angle was also presented. Its checks filled the entire 5-deg field.

Three human Ss participated in the study, two with normal vision and one wearing corrective lenses. The cornered and rounded conditions were run separately. Each session consisted of two presentations of the standard checkerboard pattern, one at the beginning and one at the end of the session, and one presentation each of the four experimental stimuli. The latter were presented in random order over sessions; the checkerboard pattern was used as an additional control for order effects.

Ss were instructed to fix their right eye on the center of the
Fig. 1. Schematic representation of the stimulation apparatus. The symbols identify the following: TRF, tungsten ribbon filament; S1, S2, and S3, circular stops, $45 \mathrm{~mm}$ in diam; $L 1, L 2$, and L3, achromatic lenses; NF, $2.0 \mathrm{log}$ Wratten neutral density filter; ST, stimulus pattern; M1, stationary mirror; M2, pivoted mirror; PM, pen motor; PS, pinhole stop; S4, circular stop, $5.7 \mathrm{~mm}$ in diam; $E$, observer's eye.

pattern. Comsweet (1956) reported that practiced Ss are able to fixate quite accurately, the eye rarely moving out of an area $10 \mathrm{~min}$ of arc in diam. Several practice fixation sessions were held before the experimental recording sessions were begun.

Four hundred responses were summed for each stimulus presentation, following which the $S$ rested while the average response was plotted. Each $S$ was run 10 times with each set of stimuli.

Each S's data were analyzed separately for both between- and within-condition differences. Between-condition differences were assessed using $t$ tests for differences between means of paired observations. In other words, for each angularly corresponding pair of corner and curve patterns, the mean amplitudes (and latencies) of the responses from each session were compared. For example, for S M.R., the means of the 4 amplitude measures from each of the 10 sessions for the 45-deg-corner pattern were compared with the means of the 4 amplitude measures from each of the 10 sessions for the 45-deg-curve patterns (i.e., 1 measurement rather than 4 from each session was used). Within-condition comparisons were done using $t$ tests for differences between pairs of individual measurements. Instead of using the mean amplitudes and latencies for each session, each measurement for a given pattern (4 per session) was compared to each measurement for each other pattern. For example, for S M.R., the 40 amplitude

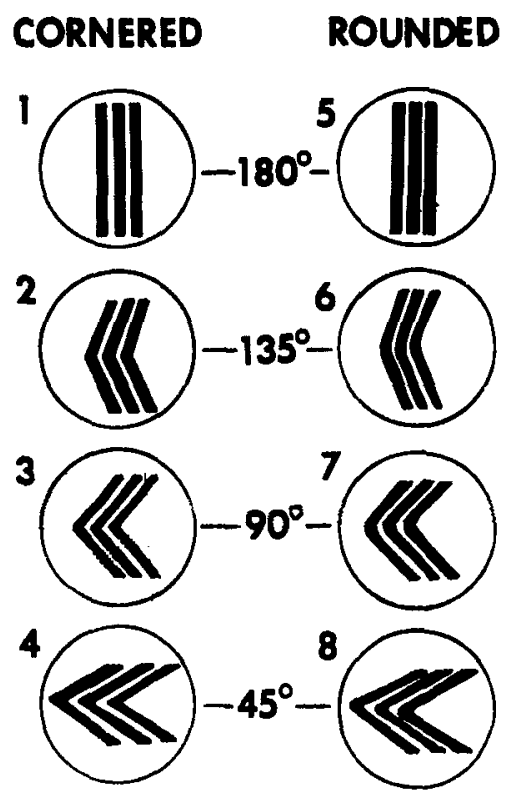

Fig. 2. Stimulus patterns as the $S$ viewed them. 
measurements for the 45-deg-corner pattern were compared with the 40 amplitude measurements for the 90-deg-corner pattern, for the 135-deg-corner pattern, and for the 180-deg pattern. A significance level of 0.05 was chosen:

\section{RESULTS}

\section{Waveform}

Figure 3 shows typical averaged waveforms for S M.R. and also the method of amplitude and latency measurement. There was little variability in waveform between sessions or between Ss. The waveforms for all three Ss were quite similar, showing a pronounced negative wave (N1), which peaked at $40-60 \mathrm{msec}$ after pattern reversal for the cornered patterns and at $55-75 \mathrm{msec}$ after pattern reversal for the noncornered (rounded and straight) patterns. This wave was followed by a prominent positive deflection (P1), peaking at $90.110 \mathrm{msec}$, and a second prominent negative deflection (N2), which peaked at 140-160 msec.

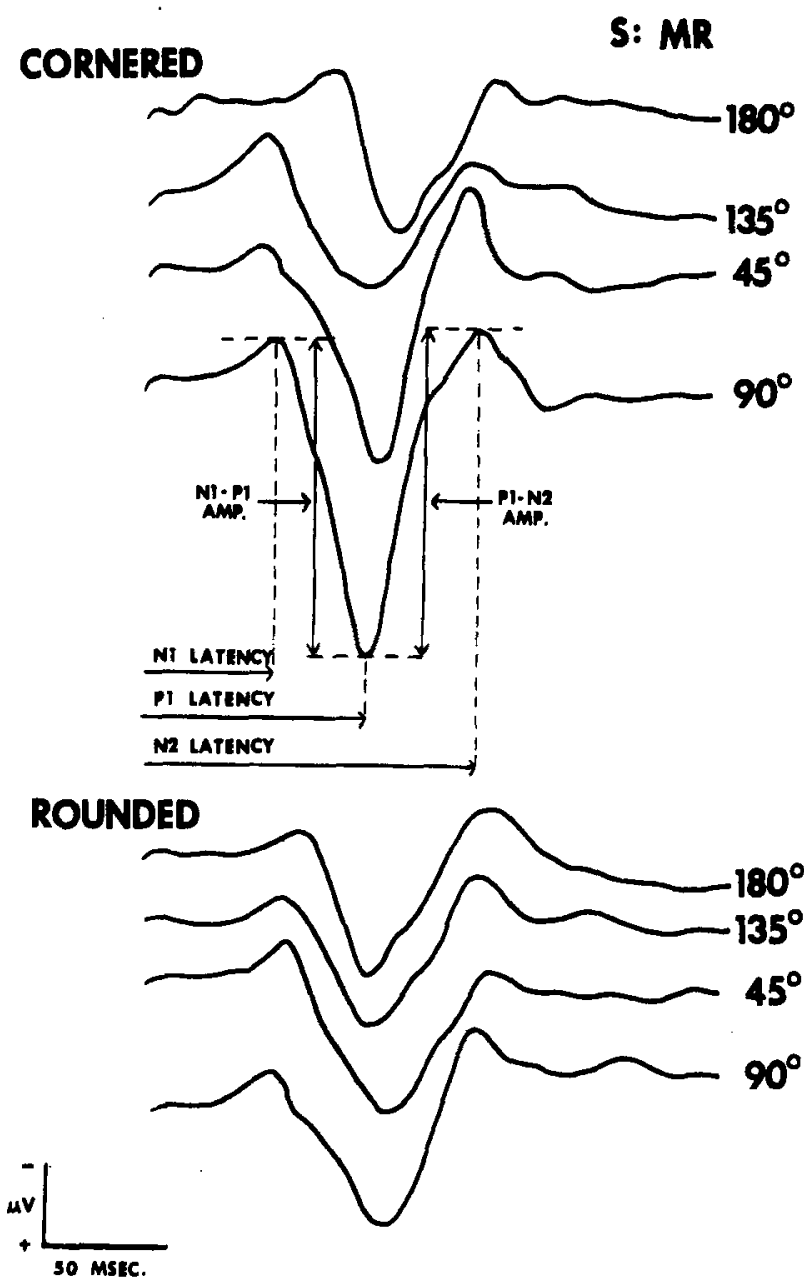

Fig. 3. Typical summated waveforms for S M.R. for each of the eight experimental stimulus patterns. Positivity at the occipital electrode produced downward deflections in the recordings. Also shown is the method of amplitude and latency measurement.

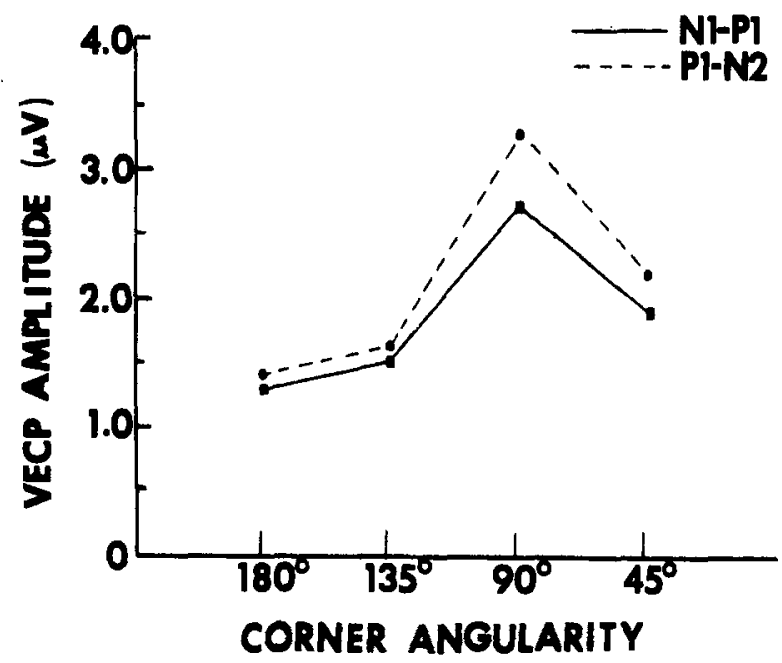

Fig. 4. VECP amplitude as a function of corner angularity for the cornered patterns for Ss M.R., D.H., and M.B. combined.

\section{Amplitude}

Several significant differences in amplitude were found. The response evoked by the 90-deg-corner pattern was of significantly greater amplitude than the responses evoked by any of the other patterns. Furthermore, for the cornered patterns, the amplitude of the response to the 45-deg-corner pattern was significantly greater than that to the $135-\mathrm{deg}$-corner pattern. The straight (180-deg) pattern evoked a significantly smaller response than any of the cornered or rounded patterns. Figure 4 shows N1-P1 and P1-N2 mean amplitudes plotted as a function of corner angularity for the cornered patterns. Figure 5 shows N1P1 and P1-N2 mean amplitude for the rounded patterns plotted as a function of curve angularity. The amplitude of the responses evoked by the checkerboard pattern presented at the beginning and end of each recording session was significantly greater than the amplitudes of the responses to any of the experimental stimuli. Response growth during each average of 400 responses was monitored on the computer oscilloscope, and response amplitude was found to increase continuously throughout the averaging period.

\section{Latency}

A major latency difference was found between patterns containing sharp corners and those without them. The latency of the initial deflection (N1) for the cornered patterns and for the checkerboard pattern was significantly shorter than $\mathrm{N} 1$ latency for the rounded and straight patterns. No N1 latency differences were found between cornered and checkerboard patterns, between any two cornered patterns, between any two rounded patterns, or between rounded and straight patterns. No latency differences between any two patterns were found for $\mathrm{P} 1$ or $\mathrm{N} 2$.

No amplitude or latency differences occurred between 


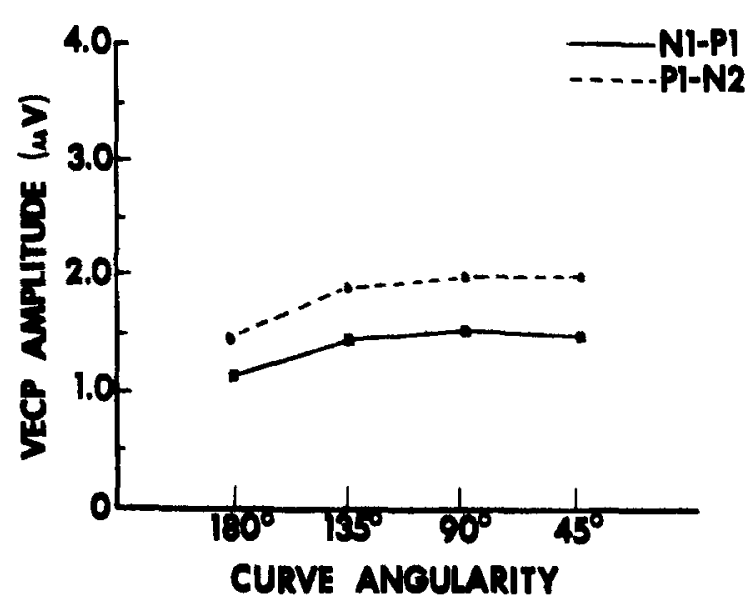

Fig. 5. VECP amplitude as a function of curve angularity for the rounded patterns for Ss M.R., D.H., and M.B. combined.

the responses to the checkerboard pattern recorded at the beginning of the sessions and those recorded at the end, ruling out the possibility of order effects, such as adaptation, habituation, fatigue, or attentional shifts.

\section{DISCUSSION}

For the three cornered stimuli, the pattern containing 90-deg-angle corners generated the largest evoked response, the pattern with 45 -deg-angle comers the next largest, and that with 135-deg-angle corners smaller; the 180-deg pattern was found to evoke a response of yet smaller amplitude. These results may be explained in terms of hypothetical retinal receptive fields with circular spatially opponent center-surround organization. We will assume that the center light bar of each pattern is centered over a receptive field of this type, since such an alignment produces a maximal response for a given stimulus. Figure 6 illustrates how a typical on-center/off-surround receptive field might respond to the three cornered patterns and to the 180-deg pattern. For all four stimuli, the excitatory center is maximally stimulated; the total amount of light falling on the inhibitory surround is also equal for all four stimuli. Hence, equal response amplitude would be expected for all patterns from a receptive field stimulated in this way. Figure 7, however, illustrates the effect of the light surrounding the outermost dark bars in each of the patterns. (See Fig. 1: note that the stripe patterns are bounded on each end by a dark bar and surrounded by a circle of light.) The amount of light falling on the inhibitory surround, and hence the amount of inhibition, is greatest for the 180-deg pattern and next greatest for the 135-deg-angle corner pattern, perhaps explaining the relative amplitudes of the responses to these two stimuli. Less light falls on the inhibitory surround for the 90-deg-angle and 45-deg-angle corner patterns. As the center-surround receptive field model would predict, the amplitude of the responses generated by these patterns exceeds that of the responses to the 135- and 180-deg patterns. However, the relative amplitudes of the responses to these two stimuli cannot be explained solely on the basis of this one receptive field model, since less light falls on the surround for the 45-deg-angle pattern, yet the 90-deg-angle pattern produces a larger response.

Neurophysiological and anatomical data indicate a predominance of horizontally and vertically oriented cortical and ganglion cell. receptive fields in cat (Pettigrew, Nikara, \& Bishop, 1968), rabbit (Levick, 1965, 1967), and man (Marg, Adams, \& Rutkin, 1968). Considering an array of simple center-surround receptive fields organized in horizontal rows and vertical columns, the superior response-evoking ability of the 90-deg-angle pattern can be explained tentatively. Figure 8 illustrates how an array of receptive fields organized in this way might respond to the three cornered patterns and to the 180-deg pattern. The light bars of the 90-deg-angle pattern fill the excitatory centers and strike only a small amount of inhibitory surround area, while the dark bars fall almost exclusively on inhibitory surrounds. Hence, the net output is relatively great. On the other hand, for the 45-deg-angle and the 135-deg-angle patterns, light bars strike large areas of both centers and surrounds, as do dark bars. Since both excitatory and inhibitory areas are being stimulated to a great extent, a smaller net response results.

White and Riggs 2 recently investigated color- and form-contingent visual aftereffects using pairs of angle patterns of complementary colors. The angles in a given pair were the same but pointed in opposite directions; across sessions, angles ranging from 60 to $172 \mathrm{deg}$ were used. White and Riggs found that the strongest angle-contingent color aftereffect occurred most often for patterns containing 90-deg angles and that speed of acquiring such aftereffects was greatest for patterns

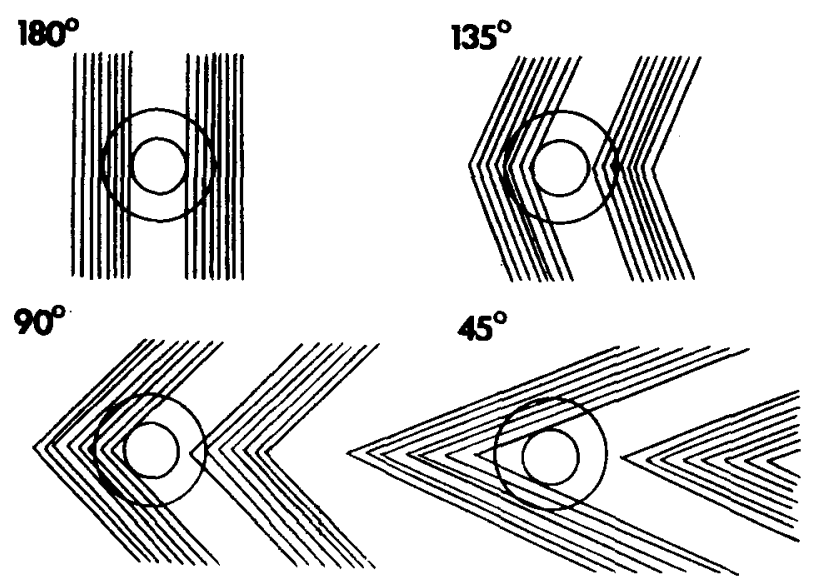

Fig. 6. How a typical circular spatially opponent receptive field with an excitatory center and inhibitory surround might respond to the three cornered patterns and to the 180 -deg pattern, considering the center light bar of each pattern to be centered over a receptive field. 
containing 90-deg and near-90-deg angles. These findings, in conjunction with our finding that the pattern containing 90-deg angles evoked the largest electrophysiological response, suggest that 90-deg angles may have some special significance for the visual system.

Both the single and multiple receptive field models fail to account for two major findings of the present study. First, based on the array model, a greater response than found in the present study would be expected from the 180-deg pattern, despite the hypothesized effects, described above, of the light surrounding the patterns. An additional factor, however, that of the possible existence of cortical orientational channels, may have contributed to the small amplitude of the 180-deg-pattern response. Campbell and Maffei (1970) found that evoked potential amplitude was greater when two or more orientations were simultaneously presented than when only one was presented, and concluded that this was due to the activation and summation of two or more orientational channels in the former case and only one in the latter. The cornered and curved patterns in the present study would have activated two channels, the 180 -deg pattern only one.

Secondly, the results for the rounded patterns cannot be explained by the center-surround receptive field formulation. On the basis of a circular spatially opponent center-surround receptive field organization, the three rounded patterns should have yielded responses of the same relative amplitudes as those generated by the cornered patterns. However, no amplitude differences were found between responses to the rounded patterns. This would seem to indicate some "special," and as yet unexplainable, effect of the presence of sharp corners on the evoked potential, such that differing angles produce responses of different amplitude for sharp but not for rounded corners.

We believe that, while no one model alone can

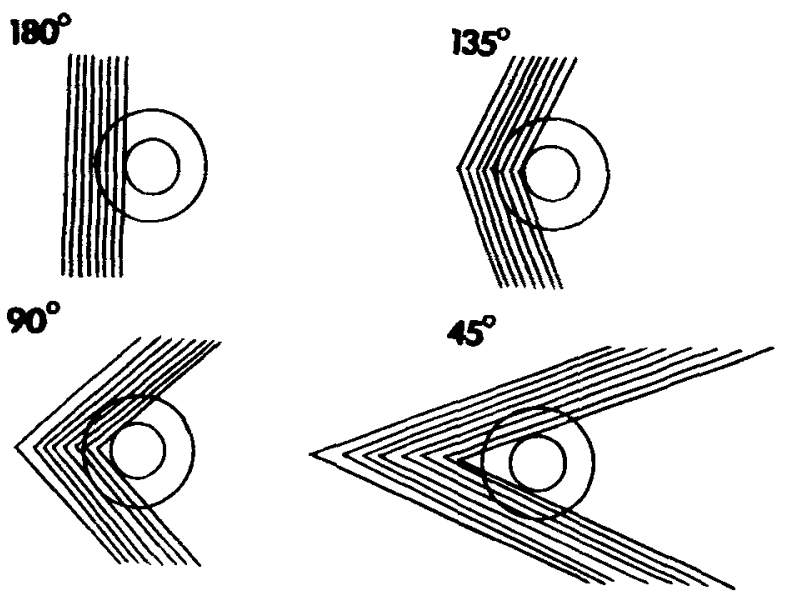

Fig. 7. Effects of the light surrounding the outermost dark bars of the cornered and 180-deg patterns on a circular on-center/off-surround receptive field.

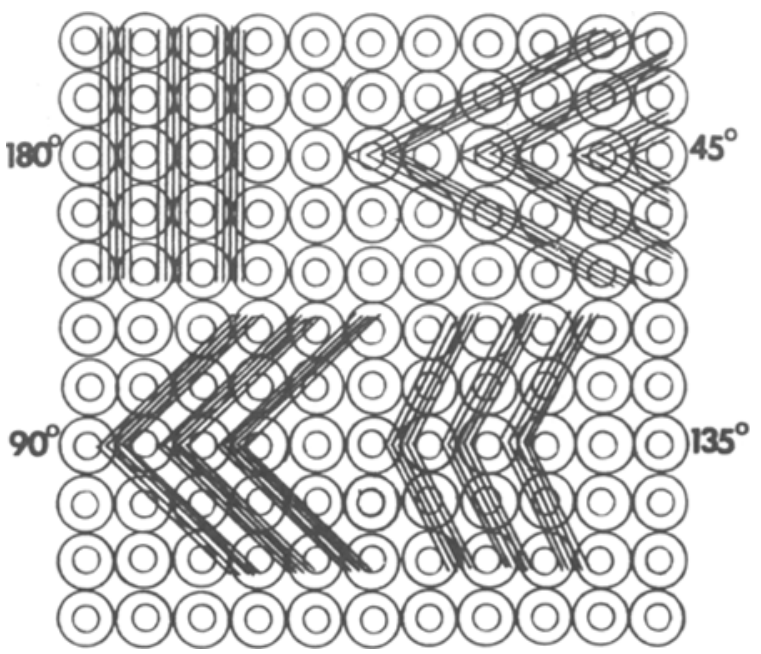

Fig. 8. Effect of the cornered and 180-deg patterns on an array of on-center/off-surnound receptive fields arranged in horizontal rows and vertical columns.

account for all of the data, the center-surround receptive field model is successful in accounting for the major portion of our findings.

\section{REFERENCES}

Armington, J. C. The electroretinogram, the visual evoked potential, and the area-luminance relation. Vision Research, $1968,8,263-276$.

Armington, J. C., Corwin, T. R., \& Marsetta, R. Simultaneously recorded retinal and cortical responses to patterned stimuli. Joumal of the Optical Society of America. 1971, 61, 1514-1521.

Campbell, R. W., Cleland, B. G., Cooper, G. F., \& Enroth-Cugell, $C$. The angular selectivity of visual cortical cells to moving gratings. Joumal of Physiology, 1868, 188, 237-250.

Campbell, F. W., \& Kulikowski, J. J. Orientational selectivity of the human visual system. Journal of Physiology, 1966, 187. 437-445.

Campbell, F. W., \& Maffei, L. Electrophysiological evidence for the existence of orientation and size detectors in the human visual system. Journal of Physiology, 1970, 207, 635-652.

Cornsweet, T. N. Determination of the stimuli for involuntary drifts and saccadic eye movements. Journal of the Optical Society of America, $1956,46,987-993$.

Gaustaut, H., \& Regis, H. Visually evoked potentials recorded transeranially in man. In L. D. Proctor and W. R. Adey (Eds.), Symposium on the Analysis of Central Nervous System and Cardiovascular Data using Computer Methods. NASA SP-72, Washington, 1964, 8-34.

Harter, M. R. \& White, C. T. Effects of contour sharpness and check size on visually evoked cortical potentials. Vision Research, 1968, 8, 701-711.

Holmes, G. Disturbances of vision by cerebral lesions. British Journal of Ophthalmology, 1918, 2, 353-384.

Hubel, D. H., \& Wiesel, T. N. Receptive fields, binocular interaction, and functional architecture in the cat's visual cortex. Journal of Physiology, 1962, 160, 106-154.

Hubel D. H. W W Wesel, T. N. Receptive fields and functional architecture in two nonstriate visual areas (18 and 18$)$ of the cat. Journal of Neurophysiology, 1965, 28, 229-289.

Johnson, E. P., Riggs, L. A., \& Schick, A. M. L. Photopie retinal potentials evoked by phase alternation of a barred pattern. Vision Research, 1966, Suppl. 1, Clinical Electroretinography, 75-91.

Levick, W. R. Receptive fields of rabbit retinal ganglion cells. American Journal of Optometry, 1965, 42, 337-343.

Levick, W. R. Receptive fields and trigger features of ganglion cells in the visual streak of the rabbit's retina. Journal of Physiology, 1967, 188, 285-307.

Marg, E., Adams, J. E.. \& Rutkin, B. Receptive fields of cells in the human visual cortex. Experientia, 1968, 24, 977-979.

Pettigrew, J. D.. Nikara. T., \& Bishop, P. O. Responses to moving sits by single units in cat striate cortex. Experimental Brain Research. $1968,6,373-390$. 
Regan. D. Evoked potentials and psychology, sensory physiology, and clinical medicine. New York: Wiley, 1972.

Reitveld, W. J., Tordoir, W., Hagenouw, J., Lubbers, J. A., \& Spoor, A. C. Visual evoked responses to blank and checkerboard patterned flashes. Acta Physiologica et Pharmacologica Neerlandica, 1967, 14, 259-285.

Spehlman, $R$. The averaged electrical responses to diffuse and to patterned light in the human. Electroencephalography \& Clinical Neurophysiology, 1965, 19, 260-268.

Spekrejise, H. A nalysis of FEG respones in man evoked by sine wave modulated lisht. The Hacue: Junk, 1966.

Timberlake, G. The visually evoked response to zig-zag patterns. Paper presented at the meeting of the American Academy of Optometry, New Yokk, 1872. Westheimer, G. The Maxwellian view. Vision Research, 1966, 6,
$669-682$.
White, C. T. Evoked cortical responses and patterned stimuli. American Psychologist, 1869, 24, 211-218.

\section{NOTES}

1. Contrast was calculated using the following formula: ( $\left.E_{1}-E_{2}\right) /\left(E_{1}+E_{2}\right)$, where $E_{1}$ is the illuminance of the light area and $E_{2}$ the illuminance of the dark area in trolands.

2. White, K. D., and Riggs, L. A. Angle contingent aftereffects of color. Personal communication, October 1973.

(Received for publication October 5, 1973; revision received November 14, 1973.) 